INTERNATIONAL DESIGN CONFERENCE - DESIGN 2018

https://doi.org/10.21278/idc.2018.0343

\title{
METHOD FOR THE DEVELOPMENT OF EARLY PROTOTYPES OF MECHATRONIC MACHINE ELEMENTS BASED ON THEIR CRITICAL PROPERTIES
}

\author{
S. Schork and E. Kirchner
}

\begin{abstract}
Prototyping in general is a widely used procedure within the product development process. Prototypes help to understand different and complex phenomena, support the communication between developers and customers and lower the risk of undesirable developments. There is however a lack of described methods for the development of the prototype itself to gain a maximum of knowledge. This paper therefore postulates a method for the development of early prototypes by identification of the critical properties of the final product. These properties lead to requirements for the prototype.
\end{abstract}

Keywords: prototyping, innovation, sensor integration, design methods

\section{Mechatronic machine elements - a radical innovation}

The integration of sensors, actuators and/or communication devices in standardized mechanical machine elements leads to the development of innovative mechatronic machine elements. Following Martin et al. (2018) the advantage of these elements is their ability to collect data in the direct vicinity of the process of interest, which minimizes and simplifies the needed calculation models as well as reducing the need for external sensors and actuators. The goal is to keep the assembly space of the mechanic machine element as well as the corresponding standardized mechanical interfaces unaltered to achieve a maximum of interchangeability. Figure 1 shows the block diagram of the mechatronic system with the use of a mechatronic machine element. The dotted lines visualize the reduced need for external sensors and actuators. Especially the integration of actuators represents a challenge because actuators often demand a higher assembly space and therefore the goal described above is harder to achieve. As a first step in the establishment phase of mechatronic machine elements, the focus is on sensor integrated mechatronic machine elements.

For mechatronic machine elements both sides, market and technology, have a high degree of uncertainty, so the development process of these innovative machine elements fits the aspects of radical innovations. Mechatronic machine elements also provide a complete set of new performance features, which is a key characteristic of radical innovations according to Leifer et al. (2006). Marketwise, the uncertainty relates to the question, if the in general higher price for mechatronic machine elements in comparison to mechanical machine elements is reasonable in relation to their higher range of functions. From the technical view, the question is, if the quality of measured variables and the precision of the control variable are comparable to these of standardized sensors and actuators. The technical side also includes questions about how to produce mechatronic machine elements in an efficient and effective way. 


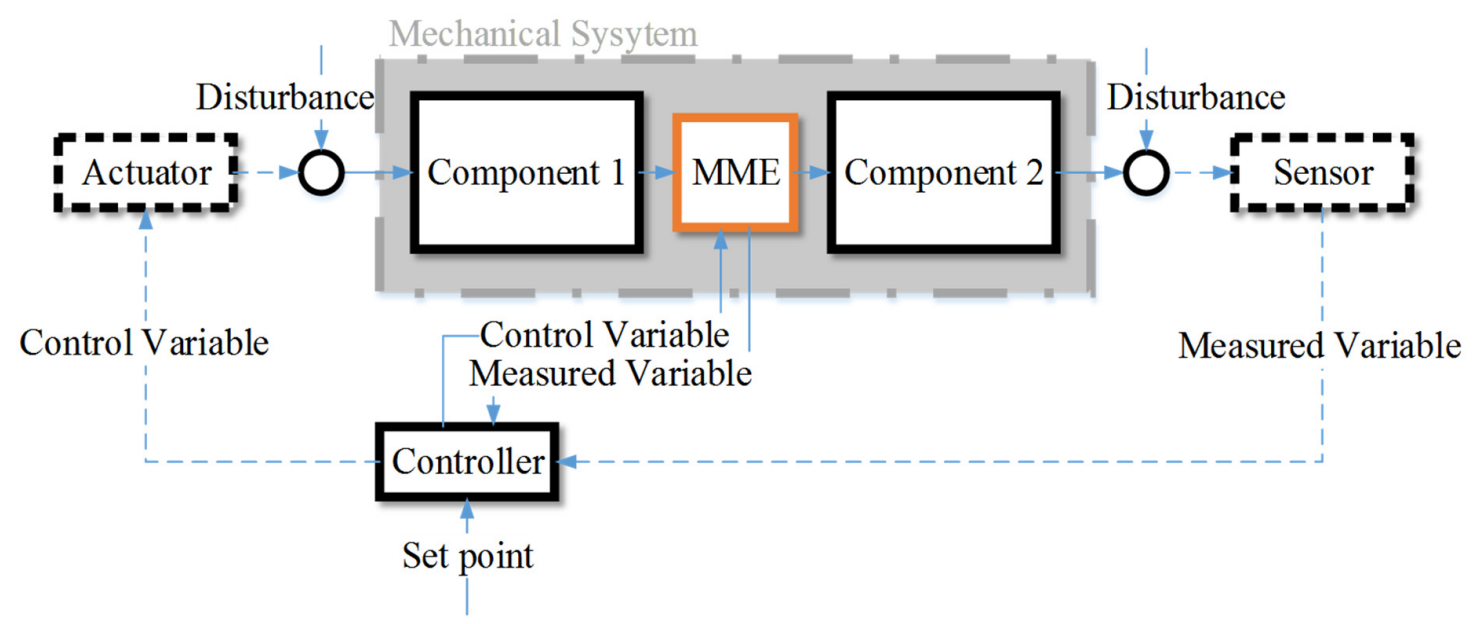

Figure 1. Mechatronic system with the use of a mechatronic machine element (based on Schork et al., 2016, the term "smart machine element" was since then changed to be more specific)

The more radical an innovation is, the less predecessor products or previous generations of the product are available for the definition of requirements or critical properties (the term is defined and explained in the later stages of this research paper). Also, the knowledge of influencing parameters and the occurrence and effects of complex phenomena is rather small. Therefore, the need for prototypes rises with the radicalness of innovations on the one hand, whereas the possibility of using predecessor and competitor products to define requirements sinks with the radicalness. Those prototypes however also entail costs in the near term of the development process, which is why the extent of prototyping activities should be minimized and the knowledge gain maximized at once. This research paper discusses the existing conflict of interest and provides a possible solution by introducing a method to identify critical properties of the anticipated final product with which the developer is capable of developing a prototype efficiently and effectively.

\subsection{Holistic product and process development of mechatronic machine elements}

The holistic product and process development model combines the process chain of the product development process with the process chain of the product life cycle. This model illustrates that the whole product life cycle causes requirements, which the developer has to anticipate. The developer on the other hand influences the product life cycle by determination of product properties, depending on the given requirements and the framework conditions of the product life cycle (Birkhofer et al., 2007).

Mechatronic machine elements may support the developer when developing new products or variants of an existing product, as shown in Figure 2. Especially the anticipation of the user behaviour is problematic. With the use of mechatronic machine elements, the developer is able to acquire information about the real user behaviour and is therefore able to customize the requirements of new products regarding the acquired information. This leads to the development of optimized products based on their application and way of usage. The developer also anticipates the different loads the product has to endure. In many cases, information about those loads are rare and/or imprecise. Mechatronic machine elements also offer the opportunity, to collect that information about occurring loads, so the developer may further customize and optimize the requirements of the new product. For example, when using a mechatronic helical beam coupling, as discussed in Section 4 of this paper, the developer collects information about the misalignment of shafts during the product use phase. Evaluation of that information may in the first place lead to the realization, that the misalignment during the use are lower than anticipated before. The developer can then adjust the requirements of the coupling in this regard and use a more cost efficient coupling for the next iteration of the product. Furthermore, that information may clarify that the load of the shaft is not as high as anticipated and therefore causing lower dynamic misalignment during the product use, so the developer is capable of optimizing the shaft design or other parts of the whole system. 


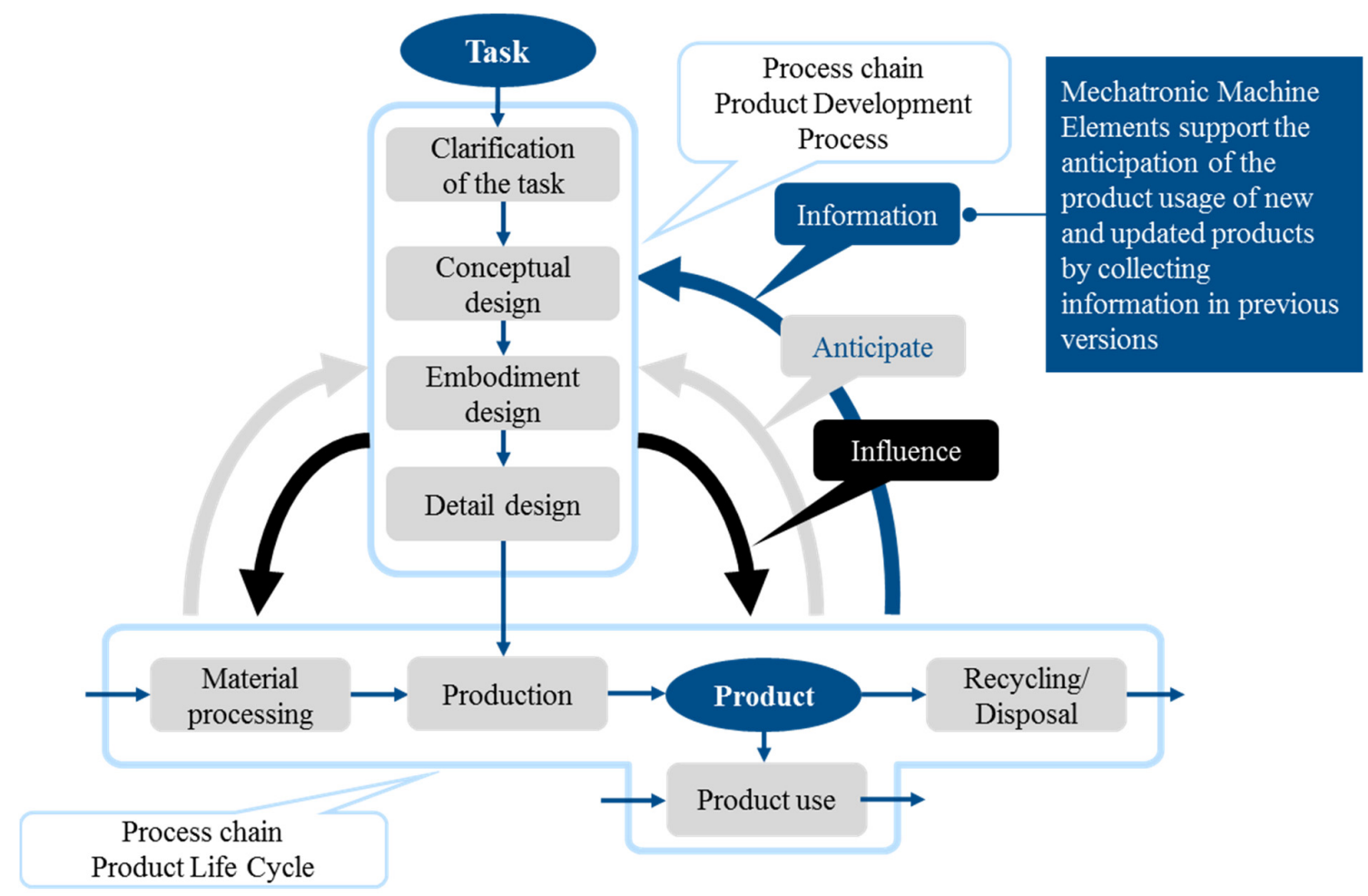

Figure 2. Holistic product and process development (Birkhofer et al., 2007)

\subsection{Definition of prototypes and their usage in the development process}

Prototypes are a major part of the product development process. Jensen et al. (2016) offer a systematic literature review regarding the different definition of prototypes. They are able to present 19 different definitions and categorize them in five distinct categories being "systematic software prototypes", "divergent prototypes", "high fidelity prototypes", "validation prototypes" and "total prototypes". These prototypes come in various forms and levels of detail, starting with virtual prototypes in form of analytic calculations and simple drawings over computer aided simulations and designs up to physical prototypes and pre-series of the final product. The literature widely acknowledges and advises the necessity of these prototypes to support the product development process and to lower the risk of failure (Faithfull et al., 2001; Elverum and Welo, 2015; Margolis, 2015; Gartzen et al., 2016). Different prototypes may serve different purposes in a large variety. This reaches from answering questions, which the development team already has, up to supporting the communication with customers and the management. For each purpose, the prototype may take another form, for example, Cooper (2002) advises that prototypes for communication with customers should be rather simple in appearance and more focused on the functions, so the customer is also more focused on the presented features of the product. This results in lowering the risk of discussions about appearance and minor design features. With each prototype, the project team is able to identify new requirements and specifications of further prototypes and the final product.

Virtual prototypes offer a maximum of flexibility but they only represent the anticipated phenomena. With physical prototypes on the other hand, the development team is able to detect unanticipated phenomena as well as complex phenomena, which are often simplified in virtual prototypes (Faithfull et al., 2001).

Figure 3 visualizes different types of prototypes depending on their state and their degree of completeness (Ulrich and Eppinger, 1995). Analytical models of single parts like shafts or models of the mass-springdamper system are very focused and completely digital (not physical). Alpha and beta prototypes on the other hand are close to the final product and thus highly comprehensive (note: The terms "alpha" and "beta" prototypes are not used consistently through literature or industry). They also advise the use of physical prototypes of single components when testing single functions of the final product. 
Hoffmann (2013) classifies prototypes in complete or incomplete and vertical or horizontal. Incomplete and complete maintain the definition as above (focused and comprehensive), whereas horizontal prototypes characterize prototypes with a broad spectrum of functions, which are rather simplified and vertical prototypes characterize those that focus an single functions but in a complex way.

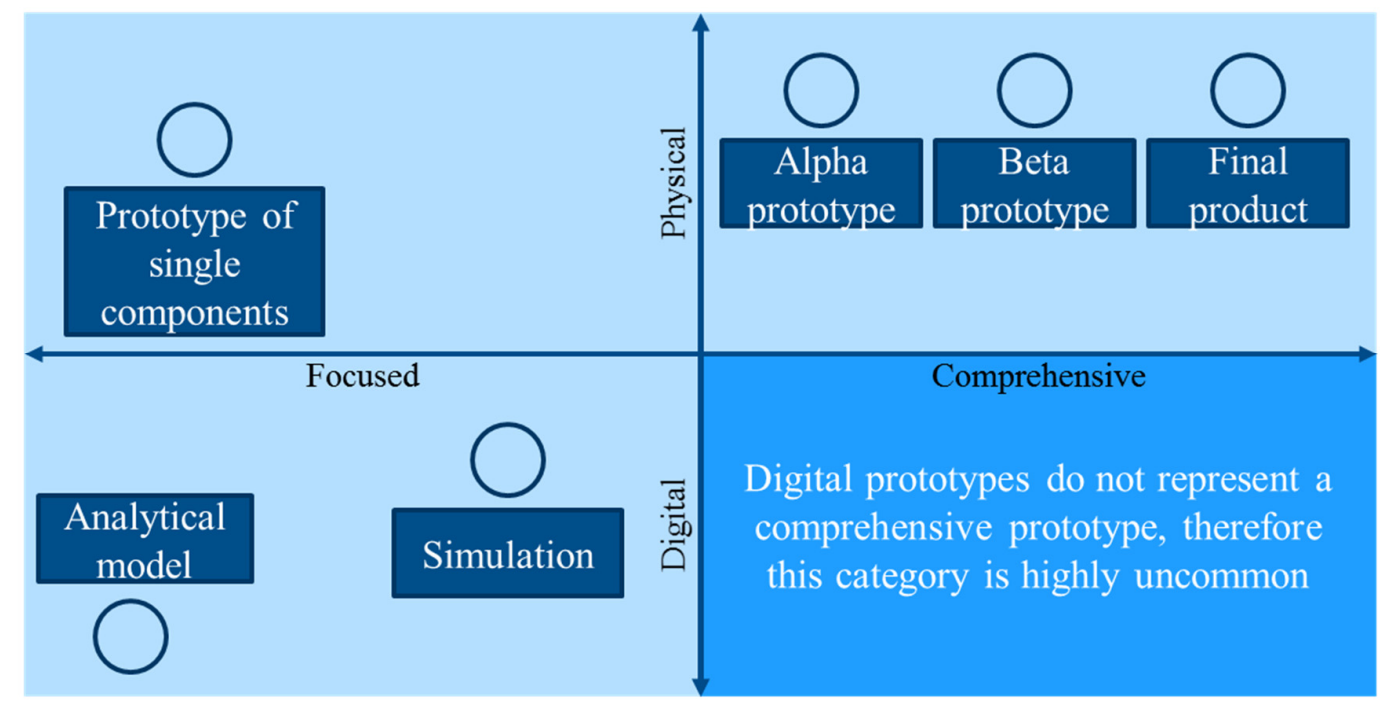

Figure 3. Visualization of different types of prototypes depending on their state (physical/digital) and their degree of completeness (based on Ulrich and Eppinger, 1995)

Producing complete prototypes is in most cases costly. Therefore, the strategy is to produce incomplete prototypes or part prototypes representing only specific areas of the final product. These prototypes are especially useful for areas of the final product, in which knowledge and understanding of processes are insufficient (Faithfull et al., 2001).

Prototypes of the product are products themselves, but the users and the use-phase are different. Therefore, the requirements for the prototype may change based on the group of users, the aspired studies and the extent of the prototype. Jensen et al. (2016) conclude that a holistic approach to prototyping strategies that include the strategic decision-making as well as the selection and application of specific prototype technologies is needed. Figure 4 depicts an example of a holistic product and process development of prototypes, based on the holistic approach of the final product (Birkhofer et al., 2007). The final product is in this case the prototype itself, for which the developer has to anticipate the variables of the product (prototype) life cycle chain. The "prototype-testing" phase substitutes the "product-use" phase and may vary with each new prototype. In this regard, the developer especially has to consider the group of users of the prototype and the defined testing procedures.

Each iteration of the prototype and subsequently each prototype-testing phase provides the development team with new information about the current state of the prototype. This enables the development team to derive new requirements for further prototypes as well as to design those further iterations.

The "material-processing" phase of the prototype may differ from the final product as well. In this regard, especially rapid prototyping is commonly referred to (Macht, 1999; Bertsche and Bullinger, 2007). In most cases, there is no necessity to manufacture the prototype the same way as the final product so in the development of the prototype the material processing and the production process of the final product are of a lower significance. Nevertheless, the developer has to take the production process of the prototype into account, especially when comparing testing results of different prototypes and concluding recommendations for the further development process.

Figure 4 also shows different types of prototypes in different stages of the product development process. In the early stages of clarification of the task as well as the conceptual design, virtual prototypes provide many advantages due to their rather low costs and fast development. Later, physical prototypes provide the opportunity, to investigate complex and rudimentarily understood phenomena. The list of different types of prototypes shown in this figure is incomplete but provides a sufficient overview. 


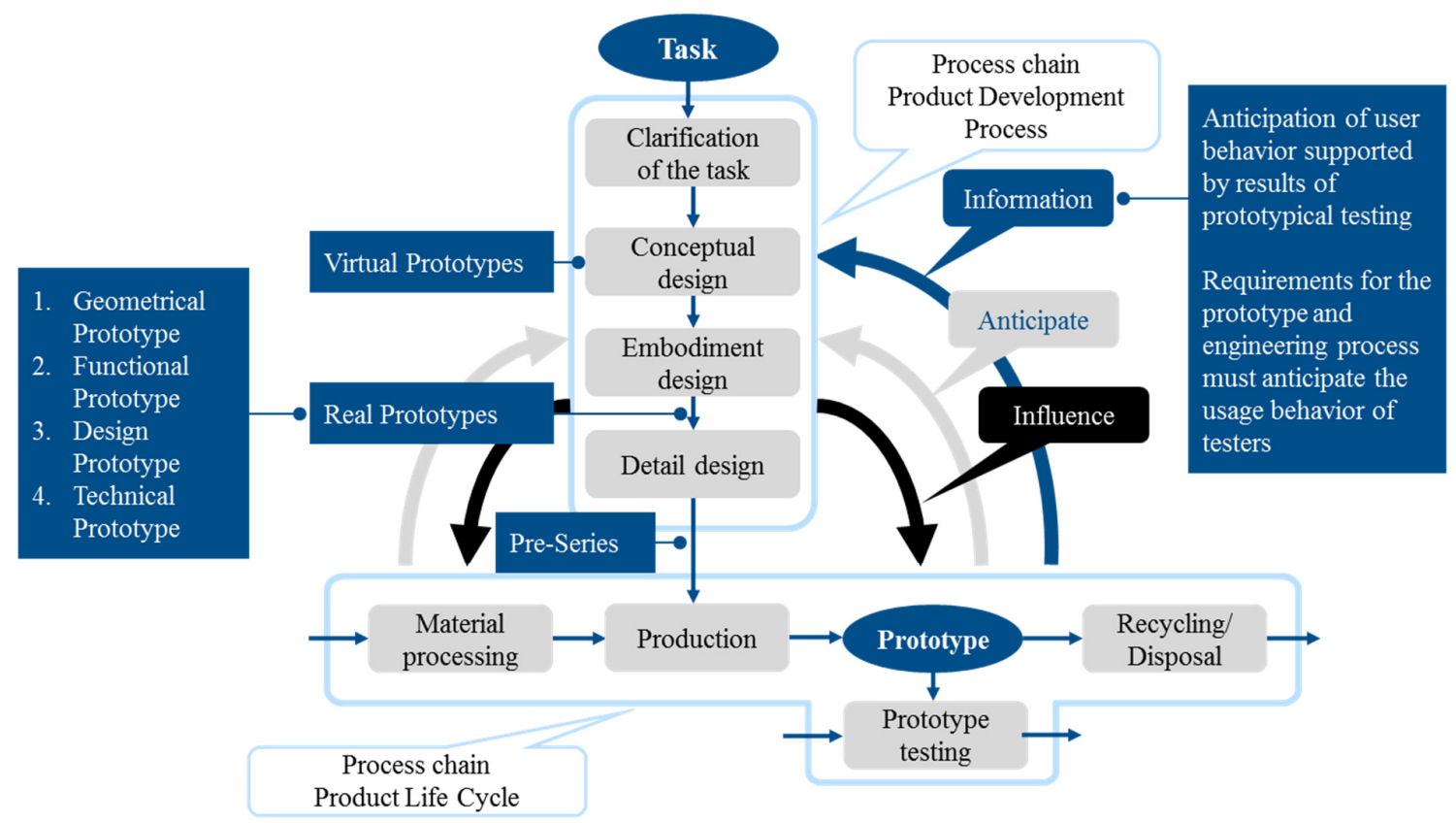

Figure 4. Holistic product and process development of prototypes (modification based on Birkhofer et al. (2007)

\subsection{The disadvantages of the current prototype development process}

A major problem in the current development process of prototypes, especially regarding radical innovations is that there is mostly no method postulated on how to develop a prototype to maximize the generated aspects of learning. Prototyping in general is therefore often intuitive and unstructured leading to an inefficient development process (Gartzen et al., 2016). Boehmer et al. (2016) point out, that prototyping is viable in every phase of the innovation process and therefore supports the agile framework for the development of physical products and they also describe iterative and creative prototyping as a core element. They therefore describe a publicly accessible and well-equipped workshop ("Makerspace") to encourage creativity and willingness for trial-and-error. Still, this process is highly intuitive and therefore susceptible to inefficiency.

Instead of discussing development methods for an efficient prototype development process, most research papers focus on different ways of manufacturing prototypes, whereby rapid prototyping predominates the discussion. Rapid Prototyping is a broadly used and explained concept. A variety of research literature discusses the advantages and benefits of being able to create prototypes in a short time. These articles focus on methods for rapid prototyping, mainly production technologies and processes as well as methods for these technologies to build the designed prototype. One topic, which is left out most of the time, is the question on how to design the optimal prototype. Each scenario offers different requirements for the prototype, whether it be packaging, haptics, acoustics or functional aspects. Camere and Bordegoni (2016) analysed various cases of prototypes of different stages and concluded that prototyping itself needs to be redefined to intensify the inclusion of the multi-layered practices and extents in prototyping.

As stated above, simplification is on the one hand a major part of the development process of prototypes to reduce development time and cost. On the other hand, oversimplification may lead to prototypes, which do not represent complex phenomena. Therefore, a conflict of interest between simplification and complexity of phenomena exists. A method for the design of prototypes therefore ideally presents a practical guideline on how to design a prototype with a low amount of resources but which offers a high amount of key insights regarding complex phenomena. 


\section{Identifying critical properties}

Faithfull et al. (2001) point out, that it is essential for the prototype that the complexity of the problem, which required the physical prototype may not be lost in the process of simplification. The focus of their paper is on scaling phenomena when developing hardware-in-the-loop-systems but this conclusion also applies to prototyping in general.

\subsection{Critical properties and requirements}

Properties in general are a notionally demarcated part of the specification of a product. They include everything that is determinable by observation, measurements, analysis, etc. of this technical system. They are of qualitative as well as of quantitative nature (Gramlich, 2013). Properties consist of a feature and a value, so each property is a combination of a feature and a value of a feature-specific value set (Birkhofer, 1980).

The term "critical" in this context includes two main characteristics. The first characteristic is a particular importance whereas the second characteristic refers to being not fully estimable. For the definition of "critical properties" the above signifies that properties, which are particular important for the correct function of the final product and/or exhibit complex phenomena are critical for the further development process, so the developer is advised to examine those thoroughly. Critical properties affect the main functions of a technical system in such a way that deviation of the value leads to major limitations of the main functions. Consequential, the final product does not represent the functions or range of functions of the initial concept.

Aside from properties, requirements take a major role in the development process. Some requirements describe product properties directly, whereas others only influence them. Regarding prototypes, the requirements partially differ from those of the final product. Table 1 shows a comparison of some requirements of prototypes and the final product. This paper only mentions the differences briefly and focusses on the critical properties but those differences are crucial for the identification of critical properties.

Table 1. Comparison of different requirements for prototypes and the final product

\begin{tabular}{|l|l|l|}
\cline { 2 - 3 } \multicolumn{1}{c|}{} & Requirement for Prototype & Requirement for Final Product \\
\hline Utilization Time & Evaluation Period & Product Lifetime \\
\hline Installation Effort & Mostly Insignificant & As Low as Possible \\
\hline Stress Resistance & Depends on Test Cycle & Mostly High \\
\hline Functional Range & Depends on Tested Functions & All Functions \\
\hline Measurability & Measurement of Tested Factors & Measurement of Factors Needed for Usage \\
\hline
\end{tabular}

Regarding stress resistance of prototypes Matthiesen et al. (2016) discuss the possibility of power-scaled prototypes and present a method to validate low load capacity prototypes. This validation method supports frontloading in the development process with the use of scaled prototypes.

\subsection{Method to identify critical properties}

The sequence of the method to identify the critical properties of a technical system postulated in this paper builds on the sequence of the failure mode and effects analysis (FMEA) as illustrated in Figure 5. The definition stage and the implementation stage subdivide the method in two stages. The definition stage includes the first steps of the method, consisting of mostly basic dissections of the system as well as the organizational part. First, the user of this method defines the system and its boundaries. In most cases, these boundaries include the complete final product and the interfaces to periphery components. Within this system, the user develops the functional structure to segment the system in subsystems and to visualize their connections. The functional structure also provides insight about energy and signal flows inside the system as well as beyond the system boundaries. After that, the user identifies and defines critical property categories and a scale to rank them amongst each other. To define those categories, the use of the method of Ehrlenspiel and Meerkamm (2017) is advised as well as regarding 
the context and the environment of the final product. The scale on the other hand builds up on the scale known from the FMEA with ranks form 1 to 10.

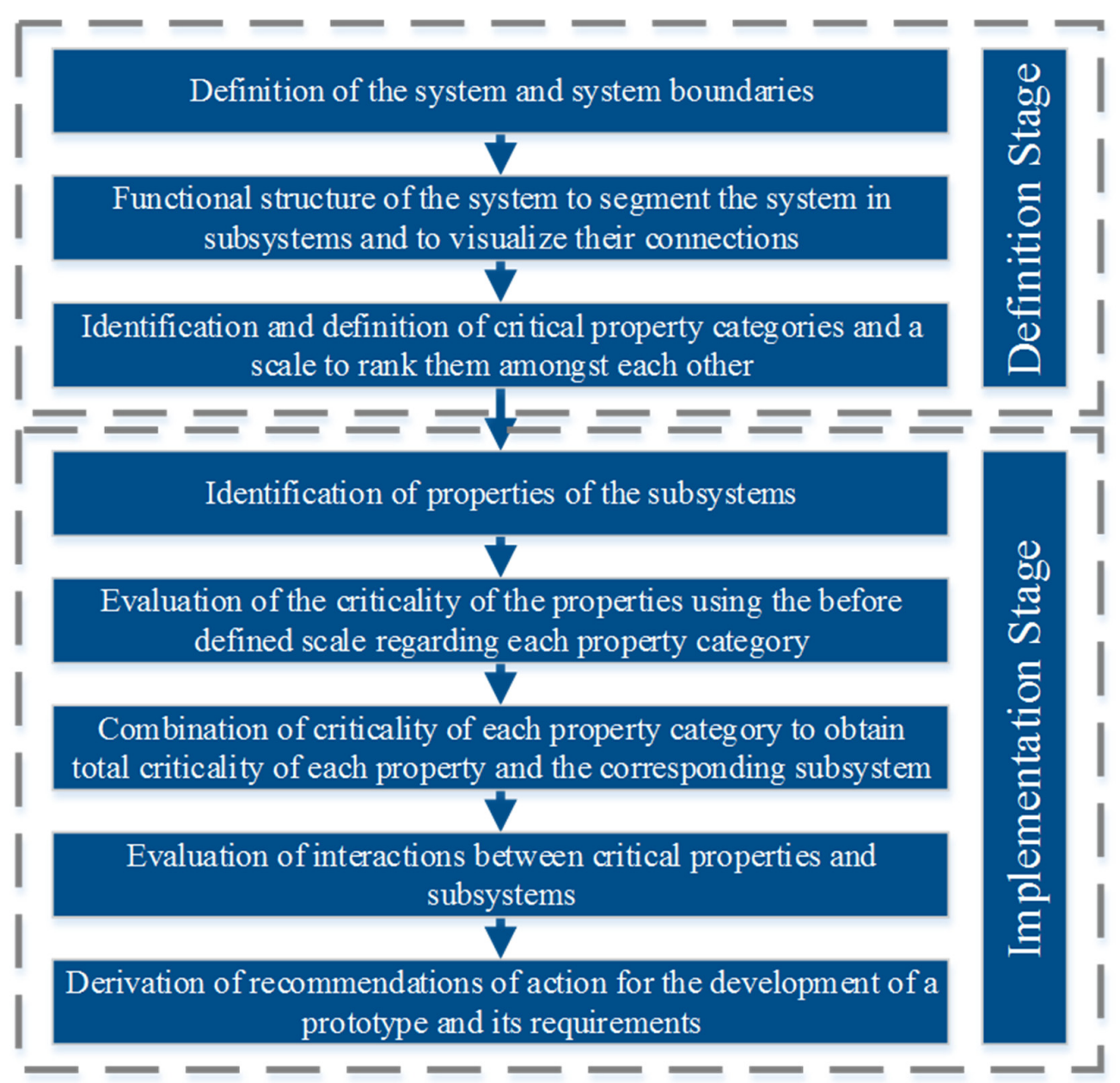

Figure 5. Identification and evaluation of critical properties of the system and its subsystem (flowchart based on the flowchart of the FMEA; DIN, 2015)

After the definition stage, the implementation stage follows. This stage starts with the identification of properties of the before defined subsystems. The user may not rate or preclude properties in this step, so an ideally comprehensive characterisation of the system is achievable. After that, the evaluation of the criticality of the properties follows. To do so, the user uses the defined ranking system and scale for each property category. Relative to the FMEA multiplying the criticality of each property category with each other leads to the total criticality of a specific property. Thereafter, the user may evaluate the critical properties regarding their connections and interactions. The last step envelopes the derivation of recommendations of action for the development of a prototype and specifically the derivation of prototype specific requirements.

\section{Abstraction of critical properties to develop an early prototype with low resources}

With the identification of the critical properties of a product, the developer is able to design a prototype to verify their effects on the final product. Especially, when the final product consists of a radical innovation, the developer may aim to verify these effects in a very early stage. To reduce costs for the prototype, it is advised to abstract the prototype and the critical properties. The essential part is to 
abstract the properties in a way they keep their characteristic features. The example of the usage of this method given in this paper shows this approach. The approach is also part of further research.

\section{Development of an early prototype of the mechatronic helical beam coupling}

Compensating couplings are in most technical systems necessary to enable the torque flow between shafts and to compensate the possible misalignment of those shafts. The mechatronic helical beam coupling is based on the idea to measure the misalignment of the shafts in-situ and in dynamic processes. The information about the current misalignment, more specifically the change in misalignment over time provides insights into forces resulting from the misalignment. Those forces in turn have an impact on the coupled system, e.g. the bearings of a motor and a transmission (Schork et al., 2016). Those effects range from influencing the operational lifetime of directly affected components up to the interference with the process causing low-quality products. Figure 6 shows the concept of the mechatronic helical beam coupling.

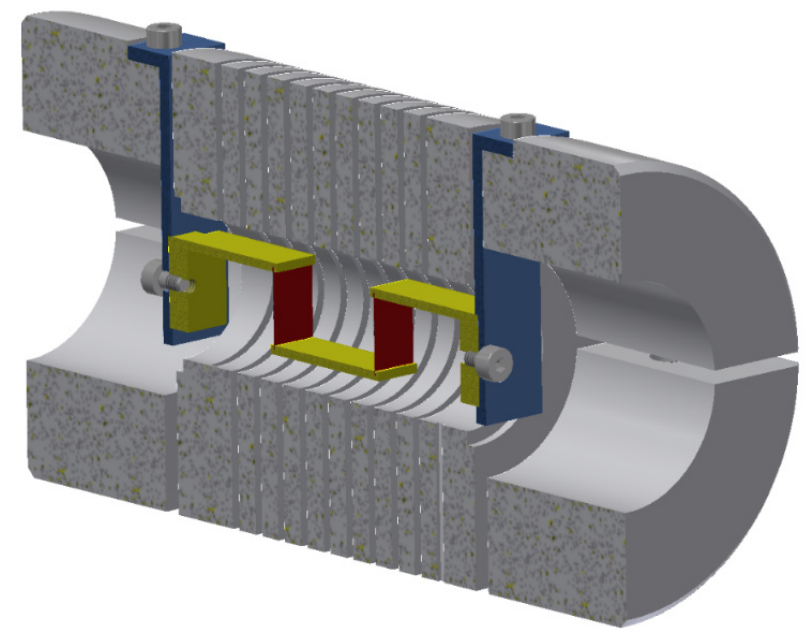

Figure 6. Conceptual design of the mechatronic helical beam coupling

The concept provides an additional element inside the coupling, which acts as a parallel path for the force flux. The stiffness of this element is rather low to prevent negative influences on the behaviour of the standard helical beam coupling. The deformation of this element caused by shaft misalignment corresponds to the deformation of the helical beam coupling. The element itself consists of two bending plates with applied strain gauges and a mount. Transferring the deformation of the helical beam coupling to the bending plates enhances the elongation of these plates on the one hand because the same amount of deformation is applied on a smaller object and on the other hand, the strain distribution becomes more describable because of the simpler geometry. Misalignment of the coupled shafts leads to a deformation of the bending plates which results in a change of resistance of the strain gauges. Depending on the characteristics of the relation of the strain gauges and the value of the measured strain, the user is provided with information about the height and the manifestation of the misalignment.

For this conceptual design, a prototype is developed using the method postulated above.

\subsection{Identification of critical properties of the mechatronic helical beam coupling}

To identify the critical properties of the mechatronic helical beam coupling, the first step is to define the system, the subsystems and the system boundaries. The system in this case includes the helical beam coupling and the additional element with the subsystems "bonding", "mount", "strain gauge", "cable" and "bending plate". Not included - and therefore out of the boundaries - are the source of energy and the data processing unit as well as the connection between those parts. After that, the functional structure 
is developed. Due to a lack of space, it is not included in this paper. To complete the definition stage, following critical property categories and a scale are defined:

- Environmental Critical: Properties in this category are affected by the environment, in which the product is used. Regarding mechatronic machine elements, especially corrosive media or currentinducing magnetic fields may cause failure.

- Function Critical: This category includes all properties, which relate to the realised functions of the concept.

- Failure Critical: Failure critical properties reference to properties, which may cause failure of the complete or partial system.

In each category, properties rated with a value between 1 and 10 depending on the severity of the criticality. In this case, the most severe failure is the deviation of the measured signal from the actual value and irreversible damage to the mechatronic helical beam coupling.

The implementation stage starts with the identification of thirteen properties of the system and with the before defined value system eight critical properties are found. Those critical properties consist of (the numbers represent the number of all properties, thus not starting with number 1):

- 4: Directing energy and signals inside the rotating system.

- 5: Linear elastic behaviour of the bending plates when under deformation.

- 6: Measurement data correspond with the anticipated values.

- 7: Strain caused by misalignment is sufficient to derive usable data.

- 9: Mount of the strain gauges provides fidelity of motion.

- 10: Mount positions bending plates exactly.

- 11: Mount does not interfere witch the measurement.

- 12: Bonding between bending plates and mount do not affect the measurement.

The next step includes the evaluation of the mechanical interactions of the critical properties. Figure 7 visualizes these interactions in their respective subsystems. This demonstrates that nearly all found critical properties influence each other. Only the cable does not interfere witch other subsystems or rather critical properties.
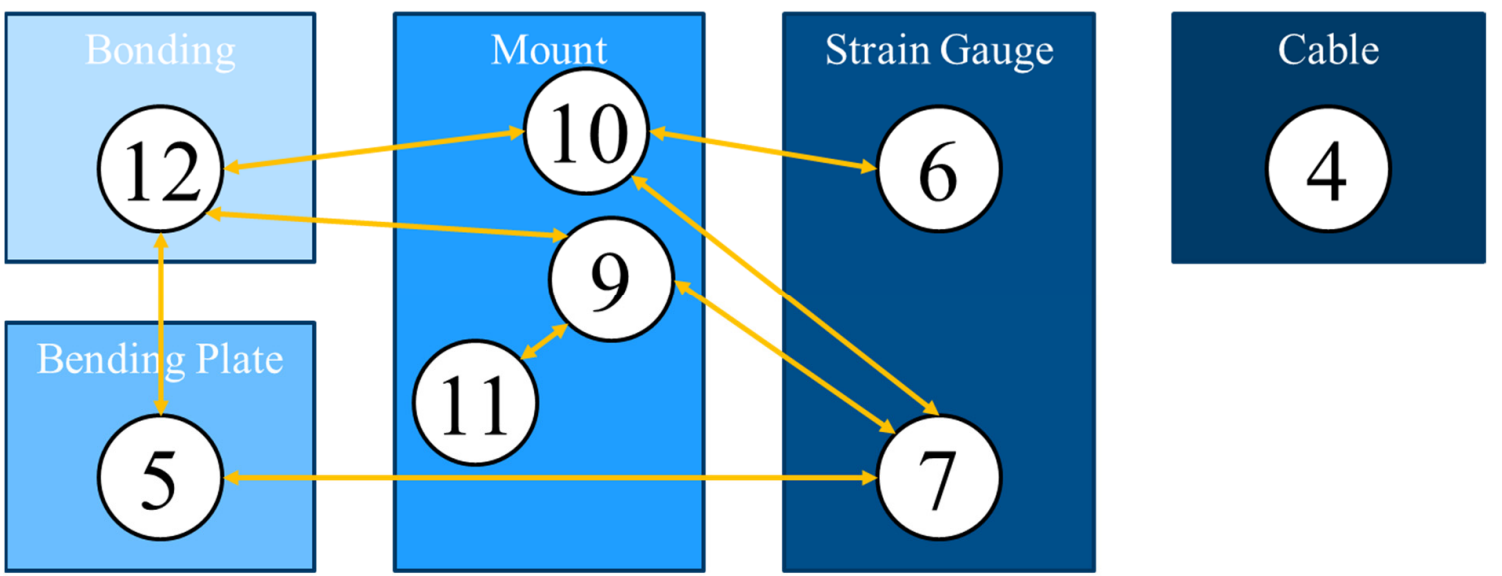

Figure 7. Visualization of interactions of critical properties in their respective subsystems

\subsection{Abstraction of the critical properties}

The final step of the implementation stage contains the derivation of action for the development of a prototype and its requirements. All critical properties are on a very basic level regarding the complexity of the phenomena and the functional extent. Therefore, the first derived recommendation is to build a basic functional prototype to verify the functionality of the concept in general. This prototype may do without the helical beam coupling and focus on the measurement element itself. Moreover, such a basic 
prototype should be tested stationary because the signal lines in and out the rotational system are very complicated to realize. Schork et al. (2016) built a first iteration of a prototype of the mechatronic helical beam coupling without the later findings. The basis of this prototype is an existing helical beam coupling. Changing the conceptual design in major ways lead to unreliable and hardly comparable testing results because the helical beam coupling itself may have contained tolerances and uncertainties, which were then transferred to the prototype. Renouncing the helical beam coupling helps to get rid of unwanted influence factors.

A second abstraction advice comes in form of the geometrical dimensions of the prototype. The concept contains a small measurement element, to fit into the helical beam coupling. With the renounce of the coupling, the measurement element may grow, making assembly easier.

The interference of the mount with the measurement element matters especially at high rotations per minute. The centrifugal forces cause the lower half of the mount outwards and therefore stretch the bending plates. This added strain may distort the measurement signal. Because it was already advised to build a stationary prototype, centrifugal forces would be of no problem. However, this critical property should definitely be investigated regarding the application of the final product in rotatory systems. The abstraction of this critical property leads to the result, that a stationary pulling force can simulate the centrifugal force resulting from high rotations per minute in a stationary system.

\subsection{Early prototype of the mechatronic helical beam coupling}

To verify the above-explained method, a prototype is developed with the results of the identification of critical properties and the derived recommendations. Figure 8 shows the extraction of the measurement element and the arrangement of the element with two shafts (left) as well as the solution for the abstraction of the centrifugal force (right). The centrifugal force is applied via a small rope and a hanging mass using the eye of the screw as fixation point for the rope and a deflection pulley not shown in this figure.

The results of these first prototypes show the general feasibility of the concept. It is possible to determine the different misalignments and the behaviour of the measurement data when rotating corresponds to the anticipated qualitative behaviour.

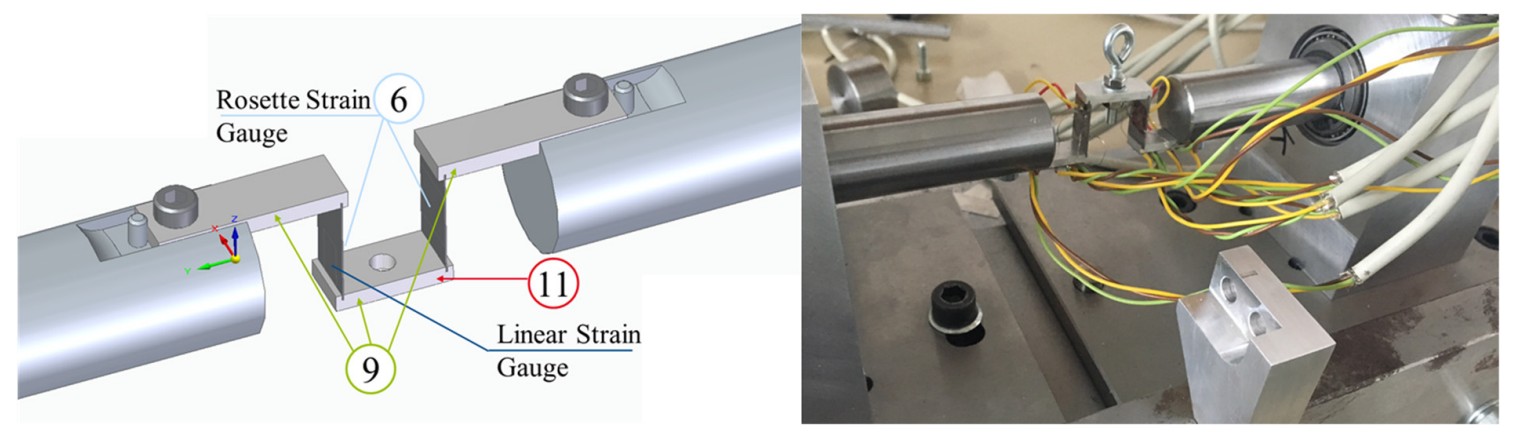

Figure 8. Extraction of the measurement element to eliminate disturbance variables (left) and preparation for the abstraction of centrifugal forces resulting at high rotational speeds by application of a stationary force caused by weight and gravity using the eye of the screw (right)

\section{Conclusion and further research}

This paper postulates a method for the developer to design an early prototype of a radically innovative product. The research results and the given example indicate the viability of the method to verify certain assumptions in the early development phases. This however doesn't mean, that the method is classifiable as overall viable. Further examples and case studies, especially regarding the usability and application in industrial practice are part of the upcoming research to improve the justification of the method. The core of this method, which is the identification of critical properties of the final product, is subject to improvement by implementing extending tools to rate the criticality of properties in an objective and universal way. 
Furthermore, the interaction between requirements of the prototype and the final product as well as between properties and requirements are a topic of discussion. Especially the last mentioned interactions open a complex field of research, which is not yet explored. Further research may discuss the derivation of prototype specific requirements to fit the needs of each prototype in each phase of the product development process. Those requirements depend on the prototype itself but also and especially on the user of the prototype. A prototype of a product in the same stage of development may have different versions for each group of testers (e.g. management, customer, user, etc.). Each prototype then produces varying testing results and the results in each group may also vary based on the heterogeneity of the individual users (Matthiesen et al., 2014). This leads to the discussion of the comparability of these different prototypes.

Another question for further research also relates to the comparability of the testing results with other prototypes on the one hand and with existing products on the other hand. Established solutions are often more refined and therefore in many cases more performant, capable or efficient. Especially early prototypes of radically innovative products and solutions may not compete with these optimized technologies. Regarding these aspects, the designer needs a method to estimate the potential of the early prototype or the solution in general even though the first testing results may lack behind established solutions. The same applies to the comparison of different prototypes, where one may be more developed than the other is, so the testing results are more accurate or one prototype is in favour based on the testing environment. It is therefore fundamental to develop a method of comparison between different prototypes and comparable established solutions.

\section{References}

Bertsche, B. and Bullinger, H.-J. (2007), Entwicklung und Erprobung innovativer Produkte - Rapid Prototyping: Grundlagen, Rahmenbedingungen und Realisierung, VDI-Buch, Springer-Verlag, Berlin, Heidelberg. https://doi.org/10.1007/978-3-540-69880-7

Birkhofer, H. (1980), “Analyse und Synthese der Funktionen technischer Produkte”, Fortschritt-Berichte VDI Reihe 1, Vol. 170, VDI-Verlag, Düsseldorf.

Birkhofer, H., Anderl, R., Franke, H.-J., Großmann, J. and Pfouga, A. (2007), "Life Cycle Engineering”, In: Krause, F.-L., Franke, H.-J. and Gausemeier, J. (Eds.), Innovationspotenziale in der Produktentwicklung, Carl Hanser Verlag, München, pp. 205-215.

Boehmer, A., Richter, C., Hostettler, R., Schneider, P., Plum, I., et al. (2016), "Think.Make.Start. - An Agile Framework", Proceedings of the DESIGN 2016 / 14th International Design Conference, Dubrovnik, Croatia, May 16-19, 2016, The Design Society, Glasgow, pp. 917-926.

Camere, S. and Bordegoni, M. (2016), "A Lens on Future Products: An Expanded Notion of Prototyping Practice", Proceedings of the DESIGN 2016 / 14th International Design Conference, Dubrovnik, Croatia, May 16-19, 2016, The Design Society, Glasgow, pp. 155-164.

Cooper, R.G. (2002), Top oder Flop in der Produktentwicklung: Erfolgsstrategien; von der Idee zum Launch, 1st ed., Wiley-VCH Verlag, Weinheim.

DIN (2015), DIN EN 60812 - Failure Mode and Effects analysis (FMEA) (IEC 56/1579/CD:2014), DIN -German Institute for Standardisation.

Ehrlenspiel, K. and Meerkamm, H. (2017), Integrierte Produktentwicklung, Carl Hanser Verlag, München. https://doi.org/10.3139/9783446449084

Elverum, C.W. and Welo, T. (2015), "On the use of directional and incremental prototyping in the development of high novelty products. Two case studies in the automotive industry", Journal of Engineering and Technology Management, Vol. 38, pp. 71-88. https://doi.org/10.1016/j.jengtecman.2015.09.003

Faithfull, P.T., Ball, R.J. and Jones, R.P. (2001), "An investigation into the use of hardware-in-the-loop simulation with a scaled physical prototype as an aid to design", Journal of Engineering Design, Vol. 12 No. 3, pp. 231243. https://doi.org/10.1080/095448201155565

Gartzen, T., Brambring, F. and Basse, F. (2016), "Target-oriented Prototyping in Highly Iterative Product Development”, Procedia CIRP, Vol. 51, pp. 19-23. https://doi.org/10.1016/j.procir.2016.05.095

Gramlich, S. (2013), Vom fertigungsgerechten Konstruieren zum produktionsintegrierenden Entwickeln: Durchgängige Modelle und Methoden im Produktlebenszyklus, PhD thesis, Technische Universität Darmstadt.

Hoffmann, V. (2013), Rapid Prototyping in der Use-Case-zentrierten Anforderungsanalyse, PhD thesis, Fakultät für Mathematik, Informatik und Naturwissenschaften, RWTH Aachen University, Aachen. 
Jensen, L.S., Özkil, A.G. and Mortensen, N.H. (2016), "Prototypes in Engineering Design. Definitions and Strategies", Proceedings of the DESIGN 2016 / 14th International Design Conference, Dubrovnik, Croatia, May 16-19, 2016, The Design Society, Glasgow, pp. 821-830.

Leifer, R., McDermott, C.M., O’Connor, G.C., Peters, L.S., Rice, M.P. and Veryzer, R.W. (2000), Radical innovation: How mature companies can outsmart upstarts, Harvard Business School Press, Boston, Massachusetts.

Macht, M. (1999), Ein Vorgehensmodell für den Einsatz von Rapid Prototyping, PhD thesis, Technische Universität München.

Margolis, D. (2015), “The Importance of Physical System Modelling to Industry. System Models That Could Have Prevented Some Costly Mistakes", IFAC-PapersOnLine, Vol. 48 No. 21, pp. 484-491. https://doi.org/10.1016/j.ifacol.2015.09.573

Martin, G., Schork, S., Vogel, S. and Kirchner, E. (2018), "MME - Potentiale durch mechatronische Maschinenelemente", Konstruktion, Vol. 70 No. 1-2, pp. 71-75.

Matthiesen, S., Gwosch, T. and Mangold, S. (2014), "Eine Methode für eine Prüf- und Validierungsumgebung zur Komponentenuntersuchung handgehaltener Geräte in der Produktentwicklung", Tagungsband 12. Gemeinsames Kolloquium Konstruktionstechnik 2014- Methoden in der Produktentwicklung: Kopplung von Strategien und Werkzeugen im Produktentwicklungsprozess, Bayreuth, Germany, October 16-17, 2014, Universität Bayreuth, Bayreuth, pp. 51-61.

Matthiesen, S., Gwosch, T., Schäfer, T., Dültgen, P., Pelshenke, C. and Gittel, H.-J. (2016), "Experimentelle Ermittlung von Bauteilbelastungen eines Power Tool Antriebsstrangs durch indirektes Messen in realitätsnahen Anwendungen als ein Baustein in der Teilsystemvalidierung”, Forschung im Ingenieurwesen, Vol. 80 No. 1-2, pp. 17-27. https://doi.org/10.1007/s10010-016-0203-Z

Schork, S., Gramlich, S. and Kirchner, E. (2016), "Entwicklung von Smart Machine Elements - Ansatz einer smarten Ausgleichskupplung", Proceedings of DFX 2016 / the 27th Symposium Design for X, Jesteburg, Germany, October 5-6, 2016, Tutech Verlag, Hamburg, pp. 181-192.

Ulrich, K.T. and Eppinger, S.D. (1995), Product design and development, McGraw-Hill, New York.

Stefan Schork, Research Associate

Technische Universität Darmstadt, Institute for Product Development and Machine Elements

Otto-Berndt-Straße 2, 64287 Darmstadt, Germany

Email: schork@pmd.tu-darmstadt.de 